\title{
UWB Vivaldi Antenna Array Lower Band Improvement for Ground Penetrating Radar Applications
}

\author{
Marc BIANCHERI-ASTIER ${ }^{1}$, Antoine DIET ${ }^{1}$, Yann LE BIHAN ${ }^{1}$, Marjorie GRZESKOWIAK ${ }^{2}$ \\ ${ }^{1}$ GeePs Group of electrical engineering - Paris, CNRS, CentraleSupélec, Univ. Paris-Sud, Université Paris-Saclay, \\ Sorbonne Université, 3 \& 11 rue Joliot-Curie, Plateau de Moulon 91192 Gif-sur-Yvette CEDEX, France \\ ${ }^{2}$ ESYCOM - EA 2552 - UPEM, ESIEE-Paris, CNAM, 77454 Marne-la-Vallée, France \\ marc.biancheri@geeps.centralesupelec.fr \\ Submitted February 1, 2018 / Accepted March 2, 2019
}

\begin{abstract}
This paper concerns a ground penetrating radar system (GPR) presenting beam forming ability. This ability is due to a great flexibility in the emission of wavefronts. The innovative concept is to use an array of antennas which can reconfigure itself dynamically, in order to focus on a desired target. This antennas system can act as a new microwave sensor to detect and characterize buried targets in an inhomogeneous medium which is the case study in various application fields such as geophysics, medical, planetology,... Its electronics are in development with the DORT (Time reversal technique) method integration for optimizing the localization of buried target. This paper aims are to present the antenna optimization used in the GPR applications. Typical antennas used in GPR are generally Vivaldi ones directly on the ground. Especially, in the context of the space mission ExoMars 2020, the radar antenna is set on a mobile station at a distance of about $30 \mathrm{~cm}$ from the ground to avoid any contact. However, they are limited by their important size, due to the lowest frequency of their bandwidth. Results of this work concern an increase of the antenna bandwidth by shifting the lowerband limit, making it a UWB type [500 $\mathrm{MHz}-4 \mathrm{GHz}]$ without changing its size. As low frequency waves can spread deeper into probed medium, this optimization can improve the radar data inversion performances.
\end{abstract}

\section{Keywords}

Ground Penetrating Radar (GPR), Ultra-WideBand (UWB), Vivaldi antenna

\section{General Description of the $\mu$ ISFINE Radar}

Ground Penetrating Radar (GPR) uses a high frequency radio signal that is transmitted into the ground and reflected signals are returned to the receiver. The principle is based on the measurement of the time taken for a pulse to travel to and from the target which indicates its depth and location. The reflected signals are interpreted for data inversion of subsurface.

The prototype aims to improve the specifications and the performance of a GPR. According to studies of GPR applications for shallow survey, the UHF frequency bandwidth considered is 1 to $3 \mathrm{GHz} \max$ [1], [2].

The key idea presented in this work is that the transmitter can reconfigure itself dynamically for focus on a bright spot (high energy zone) automatically detected by means of a previously recorded acquisition. This reconfiguration is possible thanks to attenuator/phase shifter couples and a time reversal technique [3], [4]. This corresponds to a signal to noise ratio optimization.

A standard radar survey generates massive amounts of data requiring a skilled operator for manual processing. Following an interpretation of the results, a decision will be taken for future acquisitions.

Our system called $\mu$ ISFINE (Micro Waveform-Agile Sensors for Fine Investigation in Noisy Environment) will configure itself to focus its radiance on a desired buried object. This focusing of energy will enhance the detection, localization and data inversion with an implementation of the RT-DORT method (decomposition of the time reversal operator) [3], [4].

For feasibility studies, a network analyzer is used to the generation of broadband signals and our focus is on the antenna system. The antenna array works in a multistatic configuration: each antenna can transmit and receive. To ensure the agility in terms of beam forming, the power of each antenna is controlled in phase/amplitude.

An UHF antenna array design study have been funded by the French agency Centre National d'Etudes Spatiales (CNES) to enhance its radiation pattern and enable the misalignment (see Fig. 1). The principle of misalignment of the beam with standard Vivaldi antennas was validated in this study [1] and will be optimized for this radar concept.

Once the simulation model validated, the current density on each cell of each antenna will be calculated and will 

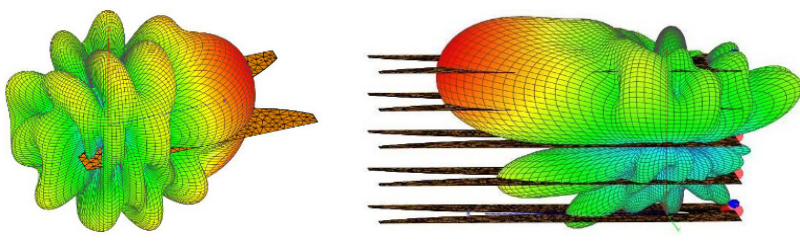

Fig. 1. Radiation patterns of the Vivaldi reference antenna with a defined footprint of $173 \mathrm{~mm} \times 299 \mathrm{~mm}$ (a), and of an antenna array made up with five reference antennas where a phase law weighting is applied (b) (a) $2 \mathrm{GHz}$ in free space.

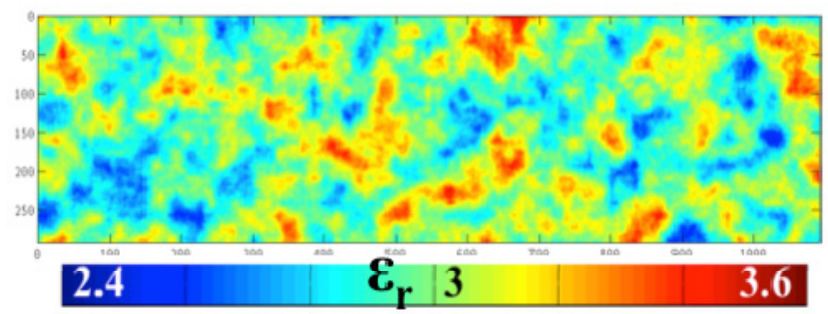

Fig. 2. An example of the fractal distribution of the permittivity (Sectional view of a modeled subsurface).

be putted directly into the TEMSI-FD software (XLIM Limoges) to modelling the antenna array behavior in a complex 3D environment (fractal distribution of the permittivity in subsurface for example [5] - see Fig. 2).

The spatial resolution of the radar is proportional to the width of the bandwidth. A balance between the bandwidth and the desired spatial resolution should be found in order not to be completely disturbed by the very small heterogeneities of the surveyed medium (neglected in this application).

Spatial resolution depends on bandwidth, directivity, aperture angle and gain. Increasing the bandwidth, only by reducing the lower frequency limit, improves the wave underground penetration and also the spatial resolution as we will see below. However, this penalizes antenna directivity, by means of the aperture angle, and consequently lowers the horizontal resolution. The use of an antenna array structure can improve the directivity and reduce this penalty.

An antenna array will be discussed in a further work because we emphasize, herein, on the localization of clefts due to the frost, which can confirm the presence of water on the surface and water ingress in the near sub-surface. Those clefts have a width of only several $\mathrm{cm}$ and are consequently hardly detectable. Previous works [6], simulations and measurements show that an aperture angle around 20 degrees is necessary for detect clefts, implying the use of an antenna array. Without the DORT method, we need at least ten reference antennas. However, using the RT technique (focusing due to the iteration) can enable us to reduce the constraint on the aperture angle, at the expense of the measurement time delay.

The transmitter signal can be achieved by a pulse or by a frequency sweeping. For a better control of the transitional regime of antennas, we develop a step frequency system. Using RT/DORT method implies to precisely know the emitted signal under the ground in the idea to reemit a part of it iteratively for focusing. An impulse radar needs to have a perfectly characterization of the transient response of the system, including antennas. In our case, the radar is a step-frequency type, in which the feeding signal is not a pulse but a continuous sinusoidal wave. Consequently, the acquisition of the surveyed medium response is achieved directly in the frequency domain, and an IFFT function enables to interpret the data in the time domain, in order to reconstruct a synthetic impulse.

In addition, for a better electromagnetic wave propagation in the near subsurface, it is interesting to shift the bandwidth lowest frequency limit as low as possible. To achieve this goal, a new HF electronics must be developed, but also a new directional antenna without increasing its size. After studies of ground electromagnetic properties, we chose to update to 0.5 to $4 \mathrm{GHz}$ (or higher).

\section{Bandwidth Optimization of Vivaldi Antenna}

\subsection{Reference Antenna:}

From a conventional Vivaldi antenna [7], [6] commonly used in GPR, we designated a reference antenna with a defined footprint of $173 \mathrm{~mm} \times 299 \mathrm{~mm}$ (less than the $200 \mathrm{~mm} \times 300 \mathrm{~mm}$ compliant with space mission size constraints - in March 2020 for example in the WISDOM (Water Ice Sub-surface Deposit Observation on Mars) context with LATMOS laboratory).

Especially, in this space mission, the radar antenna is set on a mobile station at a distance from the ground of about $30 \mathrm{~cm}$ to avoid any contact when the rover is moving (stones, hillside...). Simulations were done in free space since a previous study has shown that the presence of the ground at a distance of $30 \mathrm{~cm}$ is not modifying the frequency characteristic of such antennas [8].

With a low-cost FR4 substrate $\left(\varepsilon_{\mathrm{r}}=3.5-4 @ 1 \mathrm{GHz}\right)$ and according to [7], to achieve these dimensions, we obtain $1.21 \mathrm{GHz}$ as minimum frequency. The performances (bandwidth and radiation pattern) of this antenna are shown in Fig. 3.

As shown on the S11 trace, we observe that the antenna is efficient only from $1 \mathrm{GHz}\left(S_{11}<-10 \mathrm{~dB}\right.$; condition for bandwidth $\mathrm{BW}$ definition), and has a $107.3^{\circ}$ as aperture angle at $1 \mathrm{GHz}$ with a gain of $5.39 \mathrm{dBi}$.

In the next parts, we will explain the optimization of this antenna in order to be operational at a frequency limit lower than $500 \mathrm{MHz}$ to higher than $4 \mathrm{GHz}$.

After the surface current distribution analysis on the antenna (see Fig. 3), we conducted several studies on slot width, length, spacing, position and the number of slots with/without passive lumped element. 

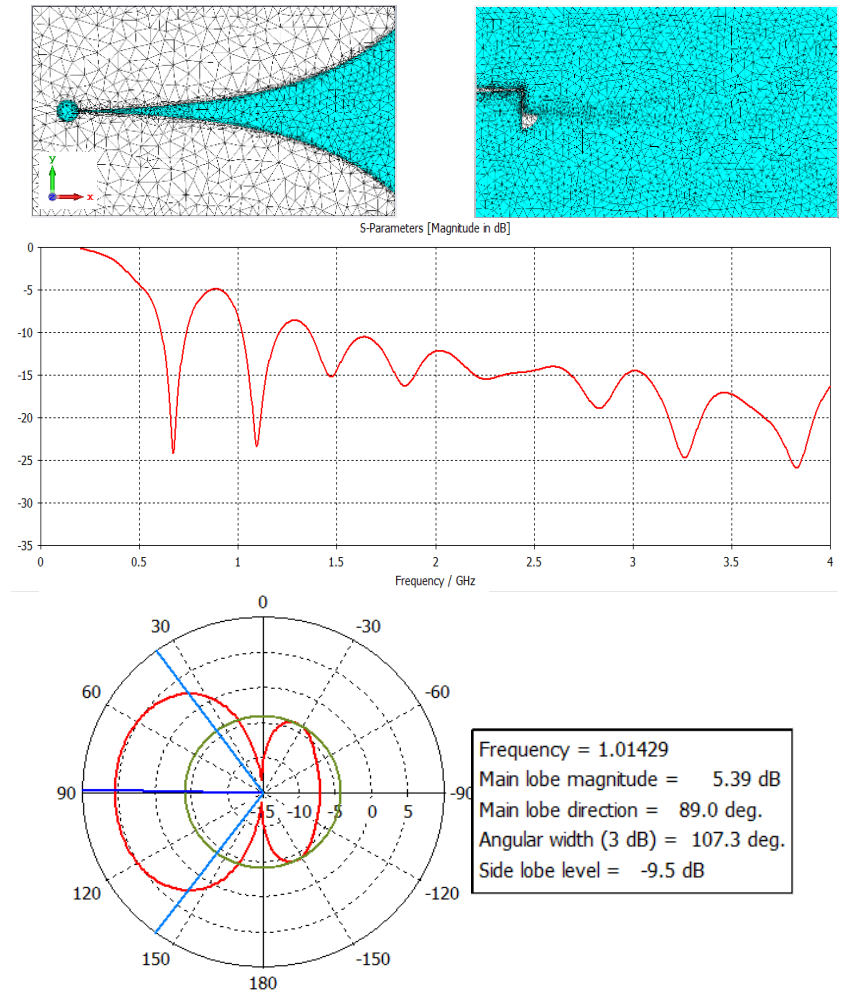

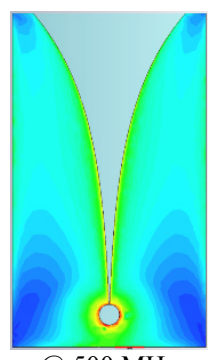

(a) $500 \mathrm{MHz}$

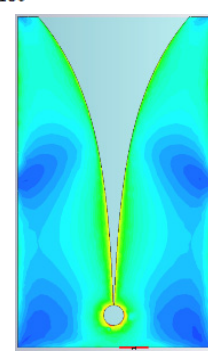

(a) $1 \overline{\mathrm{GHz}}$

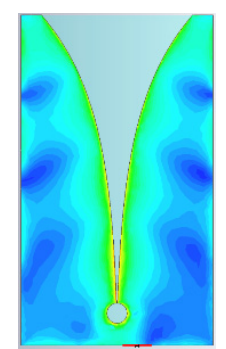

(a) $2 \widehat{\mathrm{GHz}}$

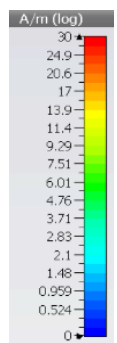

Fig. 3. Design (and mesh), reflection coefficient (S11), radiation pattern ( $\mathrm{Phi}=0^{\circ} / \mathrm{E}$ Plane)@ $1 \mathrm{GHz}$ and surface current distribution, of the reference Vivaldi antenna (antenna \#A).

The addition of slots is a very efficient improvement method [9], [10], especially when the current distribution on the antenna is studied to carefully position these disturbing slots, mainly on the outer edge of the antenna, where the return current is low.

\subsection{Optimization \#1: One Slot with Variable Length}

The key idea is to improve the bandwidth low limit by mean of increasing the path length of the current distribution as illustrated in Fig. 4.

We observe (see Fig. 5) that the slot improves the low frequency bandwidth by shifting the lower limit from $1.4 \mathrm{GHz}$ to $1.05 \mathrm{GHz}$. Nevertheless, the length (LV parameter) of this single slot has very little influence on the S11 due to the choice of low current density on the antenna

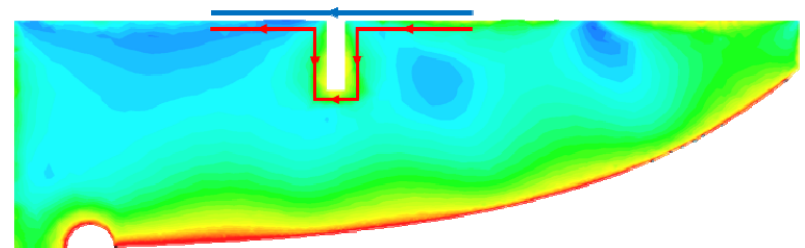

Fig. 4. Path length of the current distribution with a slot.
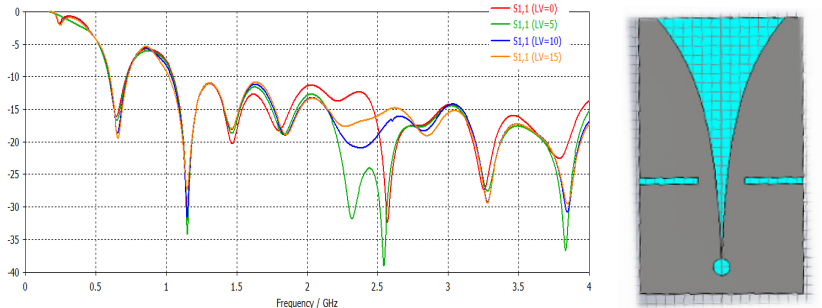

Fig. 5. Reflection coefficient (S11) and design of the antenna \#B (one slot).
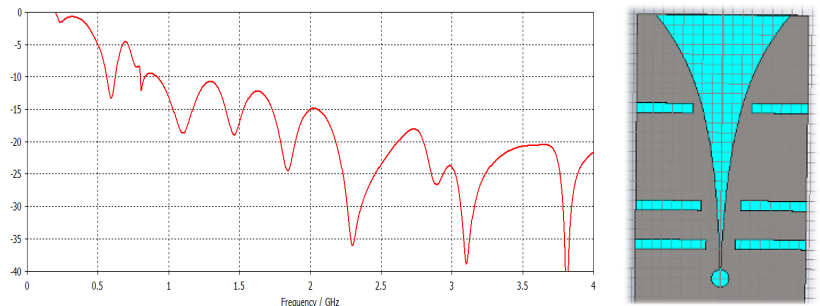

Fig. 6. Reflection coefficient (S11) and design of the antenna \#C with three slots.

surface. This drives us to continue the optimization processed by placing other slots.

\subsection{Optimization \#2: Three Slots in Fixed Position}

This antenna composed of three slots provides interesting performances with a new improvement of the low frequency of the bandwidth: from $1.4 \mathrm{GHz}$ to $900 \mathrm{MHz}$ (see Fig. 6).

\subsection{Optimization \#3a: Six Slots in Fixed Position}

This antenna, presenting six slots, provides very interesting performances with another significant improvement of the low frequency of the bandwidth: from $f_{\min }=1.4 \mathrm{GHz}$ to $750 \mathrm{MHz}$, and also with a better $|\mathrm{S} 11|$ value $(-11.5 \mathrm{~dB} @ 750 \mathrm{MHz})$, while retaining high frequencies $(>4 \mathrm{GHz})$.

We observe (see Fig. 7) an increase in the gain: from $5.39 \mathrm{dBi}$ for a standard Vivaldi antenna to $7.46 \mathrm{dBi}$ for this antenna \#3a (@1 GHz), while reducing the back lobe (from $-9.5 \mathrm{dBi}$ to $12.8 \mathrm{dBi}$ ) but losing some directivity (from $107.3^{\circ}$ to $125.1^{\circ}$ ). 

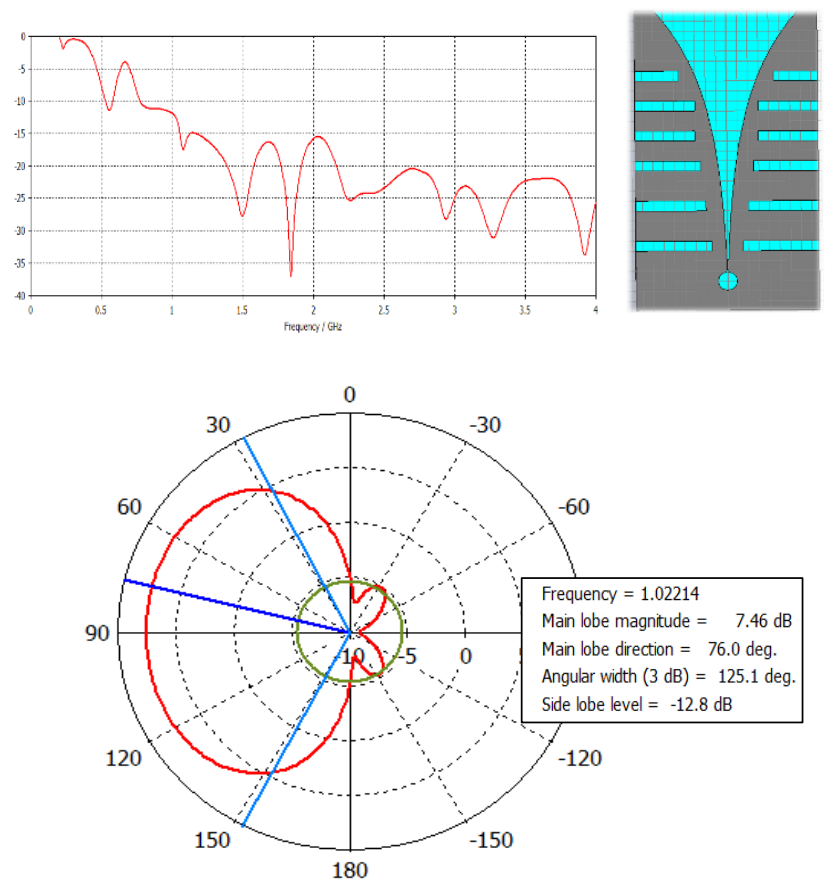

Fig. 7. Reflection coefficient (S11), radiation pattern and design of the antenna \#D with six slots@1 GHz.

\subsection{Optimization \#3b: Six Complex Slots with Modification of Cavity}

After a surface current distribution analysis, another optimization is to modify the shape of the circular cavity (at the beginning of the exponential slot) and adding disturbing patterns at the end of slots (see Fig. 8).

In Fig. 9, we still see a slight improvement of bandwidth (from $750 \mathrm{MHz}$ to $730 \mathrm{MHz}$ ) with an emission performance improvement (S11 from $-11.5 \mathrm{~dB}$ to $-13 \mathrm{~dB}$ @ $750 \mathrm{MHz}$ ). This improvement is not very significant, but it will have its interest in the next optimizations.

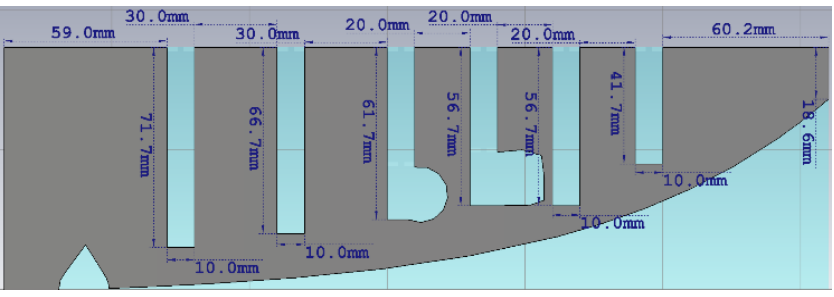

Fig. 8. Dimensions of the complete structure with slots.
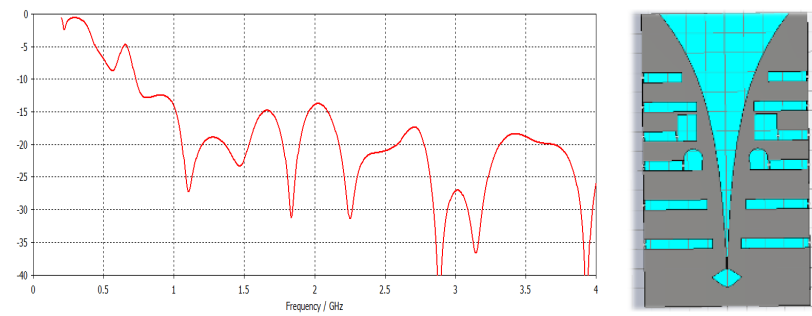

Fig. 9. Reflection coefficient (S11) and design of the antenna \#E with six special slots.

\subsection{Optimization \#3c: Six Complex Slots with Lumped Resistors}

Some antenna optimizations are realized by changing the resistive profile of conductor (Wu-King [11], [5]) optimizing the bandwidth mainly for long dipoles. In this context and after several tests, we decided to add lumped resistors. We concluded that the optimal value is $R=$ $500 \Omega$, the location of the resistors being illustrated in Fig. 10 (in blue).

Adding $500 \Omega$ resistors slightly impacts the gain of the antenna (see Fig. 10) but significantly increases the bandwidth (from $f_{\min }=730 \mathrm{MHz}$ to $470 \mathrm{MHz}$ ), while improving the antenna directionality (from $125.1^{\circ}$ to $119.3^{\circ}$ ). The gain reduced from $7.46 \mathrm{dBi}$ for (antenna without resistors) to $6.86 \mathrm{dBi}$ (antenna with lumped resistors). Simulated radiation patterns at different frequencies and the variation of simulated gain in function of the frequency are presented in Fig. 11 and Fig. 12. Typically, at low frequencies, the antenna behaves like a dipole, and it becomes more directive in the high frequencies. In Fig. 12, we can see that the gain of the optimized antenna is improved for the frequencies below $2 \mathrm{GHz}$ and is slightly degraded beyond, despite the optimization of its bandwidth and the presence of the localized resistances.
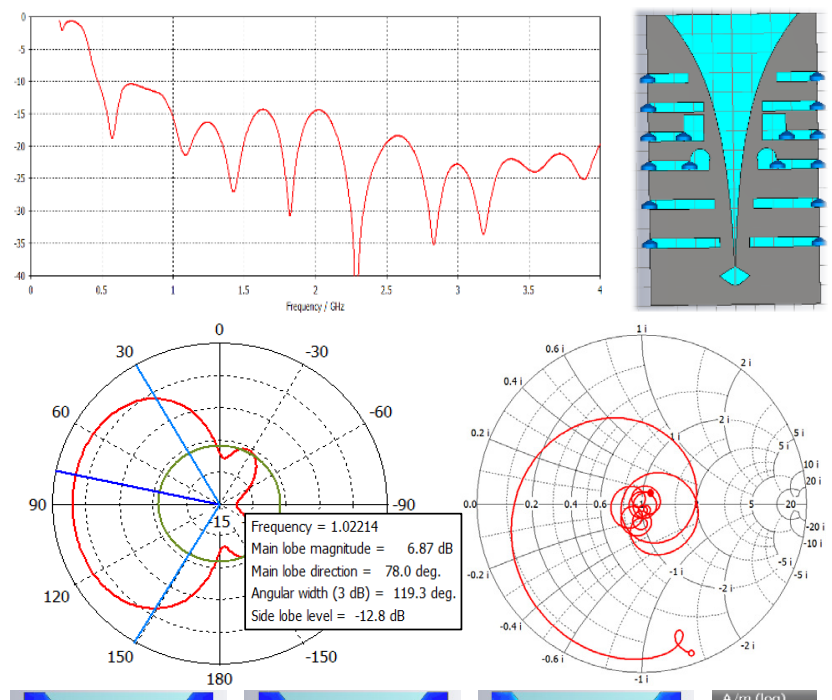

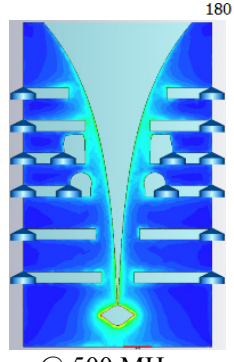

(a) $500 \mathrm{MHz}$

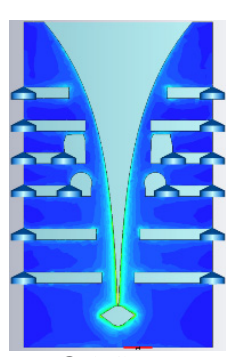

(a) $1 \mathrm{GHz}$

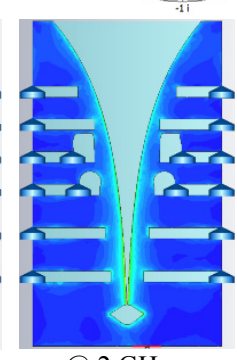

(a) $2 \mathrm{GHz}$

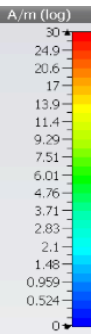

Fig. 10. Antenna \#F with six special slots with lumped resistors: design of the antenna $\# \mathrm{~F}$, reflection coefficient of the antenna $\# \mathrm{~F}$ (S11), radiation pattern of the antenna \#F (polar/3D), Smith chart of the antenna $\# \mathrm{~F}$ at $1 \mathrm{GHz}$ and surface current distribution of the antenna $\# \mathrm{~F}$. 

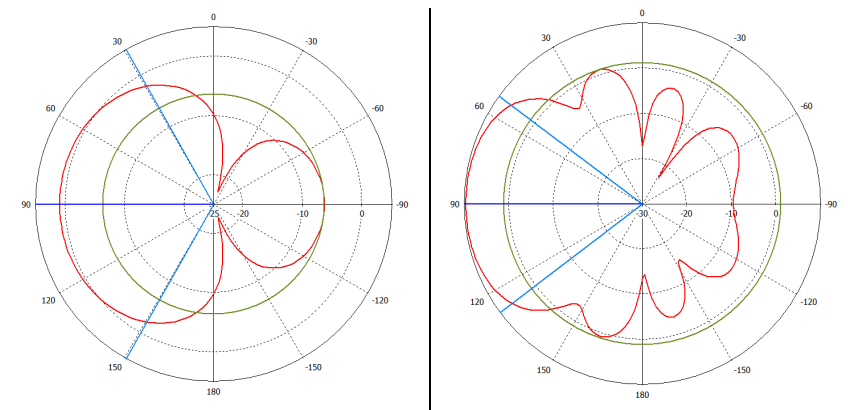

(a) $500 \mathrm{MHz}$

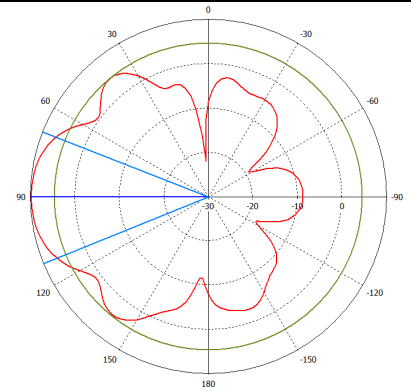

(a) $3 \mathrm{GHz}$

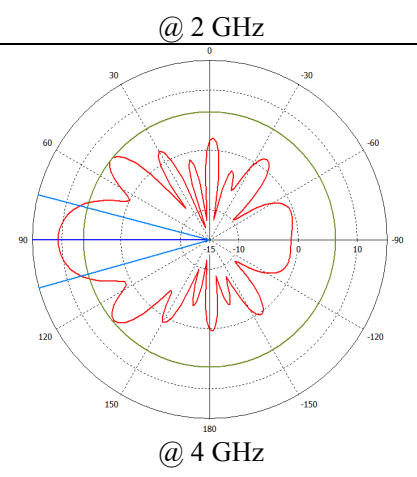

Fig. 11. Radiation pattern of the antenna \#F with six special slots with lumped resistors @500 MHz, 2 GHz, $3 \mathrm{GHz}$ $4 \mathrm{GHz}$ (@1 GHz in Fig. 10). In Fig. 12, the values of gain were plotted for all the bandwidth.

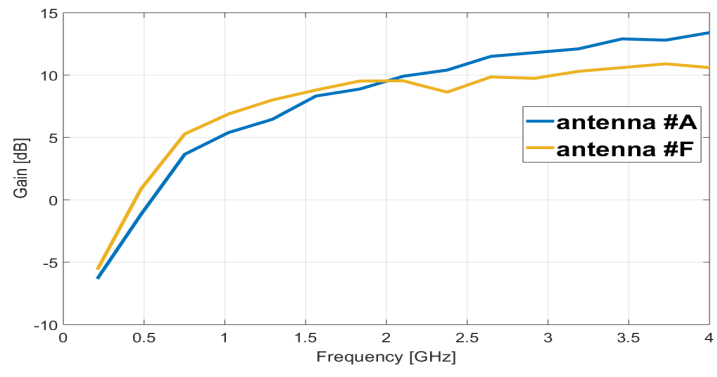

Fig. 12. Variation of simulated gain in function of the frequency $\left(\varphi=0^{\circ} \theta=90^{\circ}\right)$.

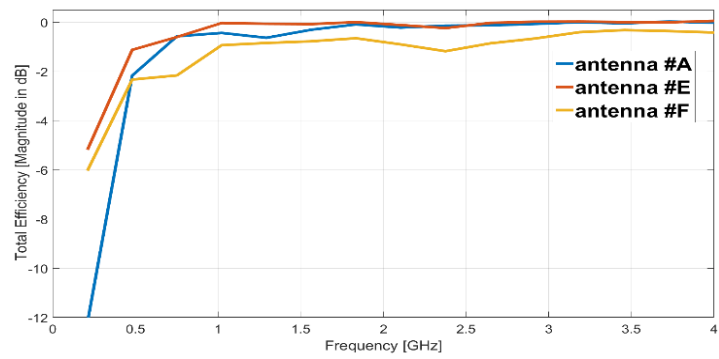

Fig. 13. Simulated total efficiency $[\mathrm{dB}]$ of the three main configurations.

The efficiency of an antenna is a ratio of the power delivered to the antenna relative to the power radiated from the antenna. Antenna efficiency is, herein, defined as the antenna total efficiency which is the product of the antenna radiation efficiency and the mismatch losses factor. A high efficiency antenna has most of the power present at the antenna's input radiated away. A low efficiency antenna has most of the power absorbed as losses within the antenna, or reflected away due to impedance mismatch. The antenna efficiency (or radiation efficiency) can be written as the ratio of the radiated power to the input power of the antenna.

Figure 13 shows the efficiencies of the previous antennas with/without slots and with/without lumped resistors. The difference is clearly marked in low frequency with an efficiency ranging from $-12 \mathrm{~dB}$ to about $-5 \mathrm{~dB}$. At low frequencies, the losses are compensated by a better adaptation. At high frequencies, losses are negligible (low current densities at resistive loads).

We have checked the polarization of the antennas. The basic Vivaldi antenna has linear (straight) polarization with study of the electric field direction (see Fig. 14), better for medium electromagnetic survey to improve the discrimination of buried targets. Despite the modifications of the antenna design, we hold a linear polarization, which is essential for the survey by georadar.

These performances are validated by measurements. As we can see in Fig. 15, the measured bandwidth in free space is from $498 \mathrm{MHz}$ to more than $5 \mathrm{GHz}$.

The UWB factor is satisfied $B W /$ fre $q_{\text {central }}>0.20$ in the lower band frequencies, unlike the IEEE standard (3-10 GHz) for telecommunications.

Experimental determinations of the aperture angle at different frequencies are presented in Tab. 1 and are in good agreement with the simulations. The measurements were done for two prototypes because one of it was encapsulated in an epoxy case, for further studies.

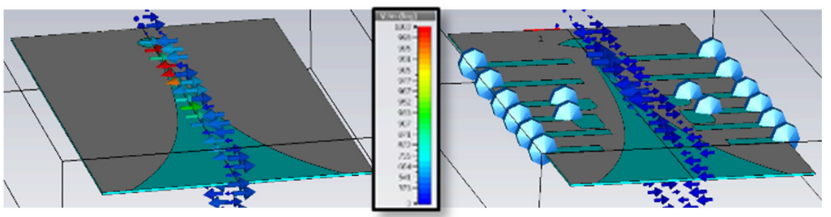

Fig. 14. Visualization of the electric field vector at $2 \mathrm{GHz}$ (at a set time) to determine the polarization of the EM wave emitted by the Vivaldi antenna.
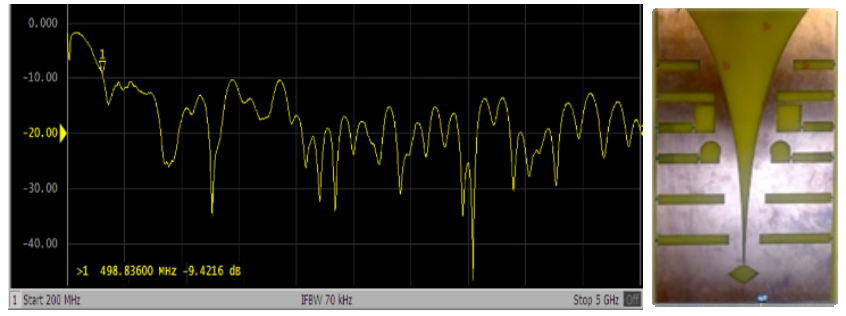

Fig. 15. Photo and measurements of the reflection coefficient (S11), of the antenna \#F with six slots with lumped resistors.

\begin{tabular}{|c|c|c|c|c|c|}
\hline & $500 \mathrm{MHz}$ & $1 \mathrm{GHz}$ & $2 \mathrm{GHz}$ & $3 \mathrm{GHz}$ & $4 \mathrm{GHz}$ \\
\hline $\begin{array}{c}\theta_{-3 \mathrm{~dB}} \\
\text { simulated }\end{array}$ & $121.2^{\circ}$ & $119.2^{\circ}$ & $73.5^{\circ}$ & $43.5^{\circ}$ & $30.1^{\circ}$ \\
\hline $\begin{array}{c}\theta_{-3 \mathrm{~dB}} \\
\text { measured }\end{array}$ & $130^{\circ}$ & $124^{\circ}$ & $76^{\circ}$ & $46^{\circ}$ & $34^{\circ}$ \\
\hline
\end{tabular}

Tab. 1. Experimental measurements of the aperture angle $\theta_{-3 \mathrm{~dB}}\left[^{\circ}\right]$ at different frequencies of the antenna $\mathrm{HF}$ (rounded average of two prototypes). 


\subsection{Optimization \#4: with Encapsulation}

At present, the objective of a low frequency of $500 \mathrm{MHz}$ is obtained. It is interesting to further reduce this low cutoff frequency in order to improve the data inversion with a better spatial resolution but above all a better wave penetration in the subsoil. At the same time, it would be useful to minimize the effect of the presence of the soil which has a variable permittivity, with a controlled environment near to the antenna, which explains our encapsulation approach.

For the following measurements with antenna encapsulated in liquid epoxy, one must expect a transposition (about 25\%) of the bandwidth to low frequencies. This encapsulation will be beneficial for our application. Nevertheless, it will bring different constraints due to the encapsulation as less heat removal, very rigid antenna, higher weight, ....

This behavior is explained by Deschamps [12] who studied the input impedance of an antenna embedded in a homogeneous conductor loss environment. Therefore, from the result of Deschamps (1), the impedance $Z_{\mathrm{c}}$ of a buried antenna in a lossy conductive medium of index $n$, at the frequency $\omega_{\mathrm{c}}$, can be deduced from its impedance in the air with a frequency shift of $(n-1) \omega_{\mathrm{c}}$, towards the low frequencies and a reduction of its level by $n$ factor.

$$
Z_{\mathrm{c}}\left(\omega_{\mathrm{c}}, \varepsilon, \mu_{0}\right)=\frac{1}{n} Z_{\text {air }}\left(n \omega_{\mathrm{c}}, \varepsilon_{0}, \mu_{0}\right) .
$$

Similarly, the bandwidth of the antenna, once buried in the medium, is shifted towards the low frequencies. The shift scale depends on the index level of the medium considered. Such approximation was already used in [13] for the measurement of WUB underground antenna.

Figure 16 confirms our approach with the measurements of the previous antenna notched in the epoxy (thickness: 4 mass side / $1 \mathrm{~mm}$ feeding side). We improve the low cut-off frequency: from $498 \mathrm{MHz}$ to $369 \mathrm{MHz}$.

\section{Conclusion and Perspectives}

As shown in this paper, we achieved the required UWB directional antenna $(0.5 \mathrm{GHz}$ to $4 \mathrm{GHz})$ to enhance the radar data inversion, without increasing the antenna original size. As a comparison, the obtained low cut-off frequency ( $\cong 500 \mathrm{MHz}$ ) corresponds to a conventional Vivaldi antenna with $600 \mathrm{~mm}$ length (corresponding to minimum half-wavelength in the substrate), unlike less than $300 \mathrm{~mm}$ in our case. Radiation pattern measurements of the designed antenna are planned in an anechoic chamber, with/without encapsulation. This encapsulation study will also target to develop isolated and buried small UWB sensors, and waterproof antennas for radar applications in extreme environments.

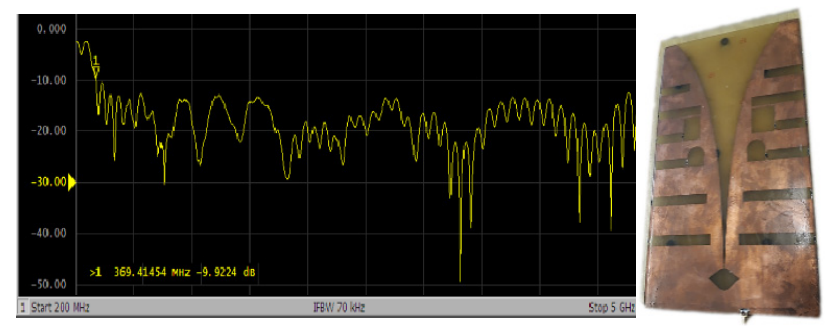

Fig. 16. Photo and measurements of the reflection coefficient (S11) of the encapsulated antenna (antenna \#G).

Currently, analytical simulations of the DORT method are completed; we must continue this study with exact numerical simulations in highly heterogeneous environment with the exact design of antennas. All the electronic cards of a radar demonstrator, allowing the control of the HF components, are finished. The RF card is under development and will be completed.

\section{Acknowledgment}

In cooperation with LATMOS (CNRS-UVSQUPMC), we have developed an antenna array to improve the specification of the planetary radar (WISDOM) selected for the ExoMars mission of ESA 2018. We thank the CNES for funding our work of network antennas.

\section{References}

[1] BIANCHERI-ASTIER, M. Study and Realization of a Mini Antenna Network in the Range UHF (1-3 GHz) - Electromagnetic Modeling of the Antenna with the Software FEKO and Magus Antenna. CNES Internal report - LATMOS-GEOPS 2013. (In French)

[2] CiARletti, V., CORBEl, C., PletTeMEIER, D., et al. WISDOM GPR designed for shallow and high-resolution sounding of the Martian subsurface. Proceedings of the IEEE, 2011, vol. 99, no. 5, p. 824-836. DOI: 10.1109/JPROC.2010.2100790

[3] PRADA, C., MANNEVILlE, S., SPOLIANSKY, D., et al. Decomposition of the time reversal operator: Detection and selective focusing on two scatterers. The Journal of the Acoustical Society of America, 1996, vol. 99, no. 4, p. 2067-2076. DOI: $10.1121 / 1.415393$

[4] FINK, M. Time reversal of ultrasonic fields. I. Basic principles. IEEE Transactions on Ultrasonics, Ferroelectrics and Frequency Control, 1992, vol. 39, no. 5, p. 555-566. DOI: $10.1109 / 58.156174$

[5] BIANCHERI-ASTIER, M., CIARLETTI, V., REINEIX, A., et al. 3D characterization of the deep sub-surface by a bistatic HF GPR operating from the surface. In American Geophysical Union - Fall Meeting 2009, id. no. \#P13B-1283.

[6] DECHAMBRE, M., SAINTENOY, A., CiARLETTI, V., et al. Wisdom GPR measurements in a cold artificial and controlled environment. In 2012 IEEE International Geoscience and Remote Sensing Symposium. Munich (Germany), 2012, p. 606-609. DOI: 10.1109/IGARSS.2012.6351521 
[7] GAZIT, E. Improved design of the Vivaldi antenna. IEE Proceedings $H$ - Microwaves, Antennas and Propagation, 1988, vol. 135, no. 2, p. 89-92. DOI: 10.1049/ip-h-2.1988.0020

[8] DORIZON, S. Preparatory Study to Interpret WISDOM Radar Data for ExoMars 2018 Mission. PhD thesis. Université ParisSaclay, France, 2016. (In French)

[9] MOOSAZADEH, M. High-gain antipodal Vivaldi antenna surrounded by dielectric for wideband applications. IEEE Transactions on Antennas and Propagation, 2018, vol. 66, no. 8, p. 4349-4352. DOI: 10.1109/TAP.2018.2840839

[10] NORNIKMAN, H., ABDULMALEK, M., AHMAD, B. H., et al. A modified antipodal Vivaldi antenna (AVA) with elliptical slotting edge (ESE) for ultra-wideband (UWB) applications. In 2017 Progress in Electromagnetics Research Symposium - Fall (PIERS - FALL). Singapore, 2017, p. 1194-1201. DOI: 10.1109/PIERS-FALL.2017.8293314

[11] WU, T., KING, R. The cylindrical antenna with nonreflecting resistive loading. IEEE Transactions on Antennas and Propagation, 1965, vol. 13, no. 3, p. 369-373. DOI: 10.1109/TAP.1965.1138429

[12] DESCHAMPS, G. Impedance of an antenna in a conducting medium. IRE Transactions on Antenna and Propagation, 1962, vol. 10, no. 5, p. 648-650. DOI: 10.1109/TAP.1962.1137923

[13] ZEMMOUR, H., BAUDOIN, G., DIET. A. Effect of depth and soil moisture on buried ultra-wideband antenna. IET Electronics Letters, 2016, vol. 52, no. 10, p. 792-794. DOI: $10.1049 /$ el.2015.3521

\section{About the Authors ...}

Marc BIANCHERI-ASTIER graduated from the University of Toulon-Var (France) and the Versailles University (UVSQ France). He received his Professional Master degree in Electronic in 2006, Research Master degree in Signal Processing in 2007, and Ph.D. (Dr.) degree in "Physical Methods in Remote Sensing" domain at LATMOS (UMR8190) in 2010. He is currently an associate professor at the Paris-Sud University (Paris Saclay) and performed his research from 2010 to 2016 in the GeoSciences Paris-Sud (UMR8148) and since 2017 is at the Electromagnetism Team in the Group of Electrical Engineering
Paris Saclay (GeePs UMR8507). His research interests are mainly focused on non-destructive survey by electromagnetic methods (GPR, UWB) for planetary subsurface/human body, and magnetic coupling (NFC/WPT).

Antoine DIET graduated from the Ecole Supérieure d'Ingénieurs en Electronique et Electrotechnique de Paris (ESIEE Paris) in 2001 (Eng.) and received the Ph.D. (Dr.) degree from the Université de Marne la Vallée in 2005 in the domain of Radio-Electronics and Telecommunications Systems. He is currently an associate professor at the Université Paris Sud and performed his research from 2005 in L2S (UMR8506) and since 2015 at the Pole Electromagnétisme in GeePs (UMR8507). His research topics concern antennas, magnetic coupling for RFID/NFC/WPT applications and wireless sensors networks (RFID-UWB).

Yann LE BIHAN is a former student of ENS de Cachan (Aggregation of Electrical Engineering in 1996). He received the Ph.D. degree of ENS de Cachan in 2000 and the Enabling Degree to Supervise PhD Studies (HDR) of Université Paris-Sud in 2007. From 2001 to 2011 he was an Assistant Professor, and since 2011 a Professor at Université Paris-Sud). In 2000 he joined GeePs (Group of Electrical Engineering of Paris). His research interests are mainly focused on characterization and non-destructive testing by electromagnetic methods.

Marjorie GRZESKOWIAK received the Ph.D. degrees in Electronics and in Microelectronics Engineering, from the Université des Sciences et Technologies de Lille (USTL) in France in 1999. In 2000, she joined the Electronic, Communication, Systems and Microsystems (ESYCOM) Laboratory at the Université Paris-Est Marne-laVallée (UPEM), as an Associate Professor. Her research interests included antenna conception, inductive coupling and millimeter wave transmission lines and filters, especially in applications to healthcare (ingestible capsule, surgical instrument traceability, retinal implant). She is currently an Associate Professor at ISAE-Supaero and her research topics concern the interactions between plasma and microwave circuits, antennas. 